\title{
Biomarkers for Evaluation of Clinical Efficacy of Multipotential Neuroprotective Drugs for Alzheimer's and Parkinson's Diseases
}

\author{
Ilan Halperin, ${ }^{*}$ Micaela Morelli, ${ }^{\dagger}$ Amos D. Korczyn, ${ }^{\ddagger}$ Moussa B. H. Youdim, ${ }^{\S}$ and \\ Silvia A. Mandel ${ }^{\S}$ \\ *Tel-Aviv Sourasky Medical Center, Department of Neurology, Memory Clinic, Tel-Aviv 64239, Israel, ${ }^{\dagger}$ Department of \\ Toxicology and Centre of Excellence for Neurobiology of Dependence, University of Cagliari, Cagliari 09124, Italy, ${ }^{\star}$ Tel-Aviv \\ University Medical School, Sieratzki Chair of Neurology, Ramat-Aviv 31096, Israel, and ${ }^{\S}$ Eve Topf Center for Neurodegenerative \\ Diseases Research and Department of Pharmacology, Faculty of Medicine, Technion, Haifa 69978, Israel
}

\begin{abstract}
Summary: During the last century, the world population has shown a staggering increase in its proportion of elderly members and thus neurodegenerative diseases like Alzheimer's disease (AD) and Parkinson's disease (PD), respectively, are becoming an increasing burden on society. Among the diverse, significant challenges facing clinicians, is the improvement of diagnostic measures to detect early and subtle symptoms, a phase in which prevention efforts might be expected to have their greatest impact and provide a measure of disease progression that can be evaluated during the course of drug treatment. At present, clinical diagnosis of $\mathrm{AD}$ and $\mathrm{PD}$ is based on a constellation of symptoms and manifestations, although the disease originated several years earlier. Given the multiple etiological nature of $\mathrm{AD}$ and $\mathrm{PD}$, it is
\end{abstract}

reasonable to assume that the initial causative pathobiological processes may differ between the affected individuals. Therefore, the availability of biological markers or biomarkers will help not only early disease diagnosis, but also delineate the pathological mechanisms more definitively and reliably than the traditional cognitive and neurological phenotypes. In the current article, we review the literature on biochemical, genetic, and neuroimaging biomarkers and discuss their predictive value as indicative for disease vulnerability to detect individuals at risk for PD and $\mathrm{AD}$, and to determine the clinical efficacy of novel, disease-modifying (neuroprotective) strategies. Key Words: Alzheimer's disease; Parkinson's disease; biomarker; neuroimaging; disease- modifying therapies; neuroprotective drugs.

\section{INTRODUCTION}

During the last century the world population has shown a staggering increase in its proportion of elderly members. All epidemiological studies show a strong correlation between age and the prevalence of Parkinson's disease (PD) and Alzheimer's disease (AD). Both $A D$ and PD are neurodegenerative disorders sharing a number of characteristics in common; both have unknown causes (except for the genetic forms), are inevitably progressive, and have a long preclinical period. In the age group of 60 to 64 years of age, the prevalence of dementia is below $1 \%$; it then doubles approximately every 5

Address correspondence and reprint requests to: Silvia Alicia Mandel, D.Sc., Eve Topf Center for Neurodegenerative Diseases Research and Dept. of Pharmacology, Faculty of Medicine, Technion, Haifa, Israel, Efron St. P.O.B. 9697, Haifa, 31096 Israel. E-mail: mandel@tx. technion.ac.il. years, reaching a prevalence of about 50\% among those age 90 years or older. ${ }^{1,2}$ As for PD, approximately 1 to $2 \%$ of the population over 65 years suffers from the disease. This figure increases to 3 to $5 \%$ in people 85 years and older. This high frequency is important in terms of social policy, because it frequently marks the need for permanent nursing care. ${ }^{3}$

Currently, a clinical diagnosis of both diseases can be made when the first motor symptoms occur, although most probably the disease originated several years earlier. Furthermore, at its initial stages, PD may be confounded by other diseases, such as essential tremor, multiple system atrophy, and progressive supranuclear palsy. ${ }^{4}$ Similar to $\mathrm{PD}$, the pathological process characteristic of $\mathrm{AD}$ begins decades before the first symptoms of brain failure, thus making it difficult to reliably identify pathology based on the clinical phenotype alone. AD may coincide with many other dementing disorders, in- 
cluding vascular dementia, dementia with Lewy bodies (DLB), supranuclear palsy and others. 5,6

The increasing prevalence of $\mathrm{AD}$ and PD motivate the drive to develop diagnostic biological markers or biomarkers (BMs) to reliably identify the pathology associated with these disorders, particularly at an early stage of the pathologic process. It is also quite possible that BMs may also help to identify subclasses of the disorders. ${ }^{7}$ Therefore, the availability of BMs for early disease diagnosis will impact the management of $\mathrm{AD}$ and $\mathrm{PD}$ in several dimensions: first, it will allow the capture of high-risk individuals before symptoms develop, a phase in which prevention efforts might be expected to have their greatest impact; second, it will help to discriminate between true $\mathrm{AD}$ or $\mathrm{PD}$ and other causes of a similar clinical syndrome. Of particular importance, a BM may help determination of the clinical efficacy of new neuroprotective therapies. The development of neuroprotective strategies is greatly hindered by the lack of markers capable of serving as surrogate objective endpoints for clinical trials testing these treatments. Among the diverse, significant challenges facing the clinicians, is the improvement of diagnostic measures not only to detect early/subtle symptoms, but also to provide an index of disease progression that can be evaluated during the course of drug treatment. Regrettably, clinical endpoints such as changes in patient's features, or the time it takes to reach the onset of specific disease-associated disabilities, may vary drastically between patients or clinical raters and require long trial durations before significant effects are seen, thus not being accurate measures of disease progression. Also, the current assessment of clinical disease improvement does not allow for a clear distinction between symptom-masking versus diseasemodifying therapies.

Neurodegeneration in PD and AD appears to be multifactorial, whereby several biochemical processes operate sequentially and/or in parallel. It is quite clear that similar or identical pathological lesions can be the consequence of multiple environmental and genetic susceptibility factors, and thus, the initial causative biological processes may differ between the affected individuals. This will have important implications as to whether identical BMs will be reflected in each etiologic subtype. Future BMs must therefore gain insight into processes underlying the pathological changes (either biochemical or regional), and act as surrogate endpoints for clinical outcomes, which would greatly improve the ability to test new disease-modifying strategies.

This review will elaborate on the available selection of biochemical, genetic, and imaging BMs for AD and PD, with a particular reference to their potential for effective early prediction and monitoring of disease progression and treatment.

\section{BIOMARKERS FOR AD}

A variety of neurodegenerative pathologies are associated with the development of late life dementia, whereas AD is considered by far the most prevalent. Sixty to $75 \%$ of all cases of dementia are diagnosed as $\mathrm{AD},{ }^{1,8}$ which is associated with progressive memory deterioration and disordered cognitive function resulting from a loss of cholinergic transmission in cortical brain regions innervated by neurones arising in the nucleus basalis of Meynert. The presence of extracellular plaques containing deposits of proteins, particularly $\beta$-amyloid, and intracellular neurofibrillatory tangles (NFTs) are hallmarks of the pathology of AD and are believed to contribute to the cognitive deficit. These may result from destructive processes involving the disruption of microtubule assembly and synaptic loss, rather than its cause. They could contribute to further neuronal damage and disease progression. ${ }^{9}$

The clinical diagnosis of demented patients is far from perfect. At present, diagnosis is based on a clinical constellation of symptoms and manifestations. To reach a consensus, diagnostic criteria have been formulated; for example, the Diagnostic and Statistical Manual of Mental Disorders (DSM) or the International Classification of Diseases (ICD) criteria, and more specific ones such as McKhann's criteria. ${ }^{10}$ BMs based on detection of pathology may diagnose the process more definitively and reliably than the traditional cognitive and behavioral phenotypes (FIG. 1). Strategies to accomplish these include, for example: 1) the detection of altered levels of tau and amyloid proteins in CSF, 2) the use of structural MRI to identify disease-specific patterns of regional or generalized atrophy, and 3) direct imaging of amyloid deposits using positron emission tomography (PET) and single photon emission computerized tomography (SPECT). At present, diagnostic markers are used extensively in the clinic (Table 1), but mainly as exclusionary markers; for example, elevated levels of 14-3-3 or tau protein in the CSF, characteristic EEG discharges and MRI signals, which suggest the existence of prion diseases "exclude" the diagnosis of AD. ${ }^{11}$

There are several reasons for the emerging need to incorporate BMs into diagnostic criteria used by clinicians to identify the pathology responsible for the patient's cognitive failure. These include: 1) the fact that a single type of pathology can produce a variety of different types of cognitive and behavioral symptoms, making it difficult to reliably identify the pathology based solely on the clinical phenotype. Even more important is the recognition that a variety of neurodegenerative and vascular pathologies can coexist, and in fact it seems that most patients with late life dementia have mixed pathologies underlying the cognitive deterioration. 2) A particular problem is whether AD can be detected at a very early stage, much before clinical criteria sufficient to diagnose dementia appear. This will become 


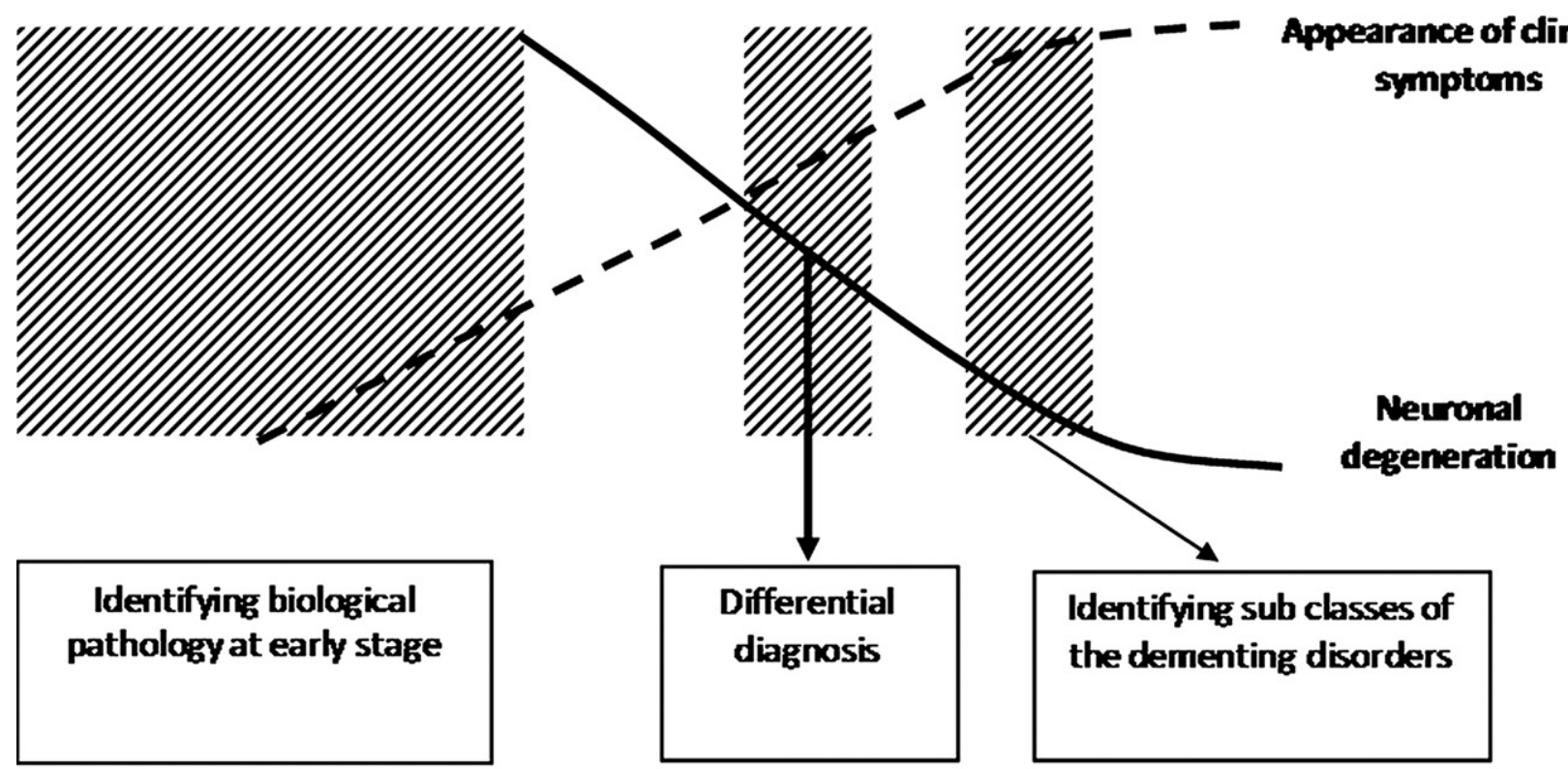

\section{Time}

FIG. 1. A model for the progressive loss of neuronal function in neurodegenerative disorders. There is a prolonged period during which loss of neuronal function has occurred, but symptoms have not yet appeared. Biomarkers may help identify this preclinical stage. At more advanced stages of the disease, when the neuronal degeneration is more severe, biomarkers can potentially be helpful in differential diagnosis and in monitoring disease progression.

especially important in cases in which treatment can achieve maximum benefit, when it is given in the earliest stages of the disease. ${ }^{12,13}$
The definition of $\mathrm{AD}$ is based on the final result, the accumulation of $\beta$-amyloid deposits and NFTs. It is quite clear that similar, or identical, pathological lesions can

Table 1. Diagnostic Value of Alzheimer's Disease Biomarkers

\begin{tabular}{|c|c|c|}
\hline Type of Method & AD Biomarker & Diagnostic Value \\
\hline \multirow[t]{2}{*}{ Genetic biomarkers } & Genes- mutations in presenilin 1 and 2 & Identify at-risk family \\
\hline & Proteins_-apolipoprotein E $\varepsilon 4$ & $\begin{array}{l}\text { Identifications of late-onset familial and } \\
\text { sporadic AD }\end{array}$ \\
\hline \multirow[t]{5}{*}{ Neuroimaging biomarkers } & MRI & $\begin{array}{l}\text { Useful in diagnosis of presymptomatic } \\
\text { and very early clinical symptomatic } \\
\text { stages of } \mathrm{AD}\end{array}$ \\
\hline & $\begin{array}{l}\text { Proton magnetic resonance } \\
\text { spectroscopy }\left({ }^{1} \mathrm{H} \text { MRS }\right)\end{array}$ & $\begin{array}{l}\text { Helpful in diagnosis of preclinical or } \\
\text { MCI stage }\end{array}$ \\
\hline & PET & Differentiation of $\mathrm{AD}$ from normal aging \\
\hline & SPECT & $\begin{array}{l}\text { Detect functional abnormalities in very } \\
\text { mild dementia }\end{array}$ \\
\hline & fMRI & $\begin{array}{l}\text { Sensitive to functional changes before } \\
\text { cognitive impairment becomes } \\
\text { clinically apparent }\end{array}$ \\
\hline \multirow[t]{2}{*}{ Biochemical biomarkers } & In serum—plasma $A \beta$ & $\begin{array}{l}\text { Elevation of plasma } \mathrm{A} \beta \text { may be helpful } \\
\text { in diagnosis of at risk asymptomatic } \\
\text { young individuals from LOAD } \\
\text { families }\end{array}$ \\
\hline & $\begin{array}{l}\text { In cerebrospinal fluid-Tau protein and } \\
\text { A } \beta \text {-peptides }\end{array}$ & $\begin{array}{l}\text { May be helpful in detecting incipient } \\
\text { AD in early MCI stage, and may also } \\
\text { be helpful in distinguishing AD } \\
\text { patients from elderly controls }\end{array}$ \\
\hline
\end{tabular}

$\mathrm{AD}=$ Alzheimer's disease $\mathrm{MCI}=$ mild cognitive impairment $\mathrm{PET}=$ positron emission tomography; $\mathrm{SPECT}=$ single photon emission computerized tomography. 
result from different causes (e.g., specific genetic mutations cause the disease in a minority of subjects, whereas in the majority $\mathrm{AD}$ is sporadic, and presumably it is the consequence of multiple environmental factors). Thus, it is not clear to what extent the initial biological processes are similar. This, of course, has important implications on the issue at hand (i.e., whether identical BM will be reflected in each etiologic subtype).

BMs can therefore be used for different purposes:

1. To identify persons "at risk" prior to disease onset.

2. To identify people at early $\mathrm{AD}$, prior to the development of the full clinical phenotype; for example, at the mild cognitive impairment (MCI) stage. Nineteen to $50 \%$ of all sufferers of MCI will develop dementia over 4 years. ${ }^{14}$

3. To differentiate AD from other neurodegenerative processes or demented individuals.

4. To monitor effects of intervention on the evolution of the disease.

5. To gain insight into processes underlying the pathological changes (either biochemical or specifically brain defined area of interest).

\section{GENETIC MARKERS OF AD}

Molecular genetic studies of neurodegenerative disorders have been useful both in identifying genes that may be causative or associated with specific diseases and in uncovering functional mechanisms subserved by products of those genes that may be markers for diagnosis or early detection. Genetic studies provide information to assess who is at risk, but except for the case of rare genetic disorders, they do not provide complete information when, or if, those identified as being "at risk" may expect the onset of neurodegeneration.

In the case of $\mathrm{AD}$, mutations in several genes are now known to cause the disorder in families with an autosomal dominant pattern of inheritance. Detection of the mutations in these genes can identify at-risk family members, but it is not generalizable to sporadic AD. ${ }^{15,16}$ In $\mathrm{AD}$, the identification of $\beta$-amyloid precursor protein and presenilin 1 and 2 mutations has strengthened the amyloid hypothesis and identified potential sites for pharmacological interventions.

An important relevant issue is the genetic association with the increased risk of developing $\mathrm{AD}$ in families that carry the apolipoprotein $\mathrm{E} \varepsilon 4$ allele. ApoE is a protein involved in the transport of cholesterol. Three apoE gene alleles are described $(\varepsilon 2, \varepsilon 3$, and $\varepsilon 4)$. A growing volume of evidence has reported an association of apoE $\varepsilon 4$, late-onset familial, and sporadic AD. ${ }^{17}$ As many as 40 to $50 \%$ of $\mathrm{AD}$ patients possess $\varepsilon 4$ allele compared to 15 to $25 \%$ of controls. ${ }^{18}$ Subjects homozygous for $\varepsilon 4$ are reported to have a 6 - to 8 -fold increased risk of developing
AD compared to the risk of heterozygotic subjects (increased by 2 - to 4 -fold $)^{17}$ and, to date, no other late onset $\mathrm{AD}$ (LOAD) gene has been conclusively proven.

Obviously, genetic markers are by themselves not sufficient to make a clinical diagnosis of AD. The mutation, if it exists, has been there since conception and birth of an individual. Thus, a person carrying a disease-causing mutation is not immune to the development of other diseases, and the diagnosis must be supported by clinical evidence.

Ultimately, sensitive methods for identifying genetic background may provide a better means for characterizing a statistical risk before disease manifestation. Nevertheless, it seems logical to assume that effective identification of the disease must combine genetic characteristics with identifying a clinical phenotype and/or additional BMs, either for early detection or for the more correct diagnosis of an already existing disease. ${ }^{19}$

\section{NEUROIMAGING MARKERS}

During the past decade, neuroimaging has emerged as a strategy to define neurodegenerative disorders' phenotype in both preclinical and early clinical disease stages. Imaging provides a window to the underlying processes in these disorders, since loss of brain volume is one of the earliest consequences of neuron death.

\section{Structural imaging using MRI}

While helpful in supporting the diagnosis of $\mathrm{AD}$, this approach is confounded by the presence of atrophy due to other pathological processes and the generalized atrophy that occurs as part of normal aging. ${ }^{20,21}$ One way to confront this problem is to use measurements of multiple anatomically specific brain volumes other then the hippocampus and entorhinal cortex that are affected by AD. Examples include: the amygdale, superior temporal sulcus, cingulate gyrus, temporal-parietal regions, and the frontal lobe. This provides the potential to increase the diagnostic power of MRI-derived atrophy measurements by additional anatomically defined areas. ${ }^{22}$ By modifying existing clinical MRI acquisition protocols to incorporate measures of $T_{1}$ relaxation time, it is possible to quantify the accumulation of abnormal protein aggregates in anatomically-defined brain regions. This opens the possibility of developing MRI-based molecular imaging BMs for AD-related pathology that could be used in healthcare facilities. ${ }^{23}$

Proton MR spectroscopy $\left({ }^{1} \mathrm{H}\right.$ MRS) is a diagnostic imaging technique that is sensitive to changes at the cellular level. With ${ }^{1} \mathrm{H}$ MRS, several of the major protoncontaining metabolites in the brain can be measured during a common data acquisition period. The metabolite $\mathrm{N}$-acetyl aspartate (NAA) is a marker for neuronal integrity. NAA decreases in a variety of neurological dis- 
orders, including clinically diagnosed AD. ${ }^{24,25}$ The decrease of NAA or the NAA/creatine $(\mathrm{Cr})$ ratio was observed in the posterior cingulate of AD patients. ${ }^{26,27} \mathrm{In}$ patients with mild to moderate $\mathrm{AD}, \mathrm{NAA} / \mathrm{Cr}$ levels are lower than normal in the medial occipital lobe, including the visual cortex. This regional pattern is in agreement with the distribution of the neurofibrillary pathology, and associated neuron loss in people with mild to moderate $\mathrm{AD}$, indicating that NAA/Cr levels are potential surrogates for early detection of $\mathrm{AD}$ and for disease progression. ${ }^{28}$ Another metabolite peak of interest in the ${ }^{1} \mathrm{H}$ MRS of the brain in AD is the choline (Cho) peak. The largest amount of choline in the brain is in choline-bound membrane phospholipids that are precursors of choline and acetylcholine synthesis. It has been postulated that the elevation of Cho peak is the consequence of membrane phosphatidylcholine catabolism to provide free choline for the chronically deficient acetylcholine production in $\mathrm{AD} .{ }^{29}$ This appears to be useful in detection of presumed pathologic changes in people with clinical diagnosis of AD. Lower, NAA levels, NAA/Cho has also been observed at the preclinical or MCI stage. ${ }^{30}$

\section{PET and SPECT}

$\mathrm{AD}$ is associated with metabolic impairments that often have a "typical" pattern, initially affecting the parietal and temporal lobes, as well as posterior cingulate cortex, before progressing to the frontal lobes and other brain structures. This metabolic activity can be visualized using ${ }^{18} \mathrm{~F}$-2-fluoro-deoxy-D-glucose PET and the pattern of involvement is often useful in distinguishing AD from other late-life dementia. ${ }^{31,32}$

The impaired metabolic activity may, in part, be due to a loss of neurons in these areas and may be a sign of reduced activation (e.g., reflecting synaptic loss). ${ }^{33}$ Evidence exists that the pattern of temporal and parietal hypometabolism and hyporeperfusion are reasonably sensitive and specific to AD. Although differentiation of $\mathrm{AD}$ from normal aging seems clinically irrelevant, the evidence for the ability of PET and SPECT to detect functional abnormalities in very mild dementia has considerable importance in the early diagnosis of dementia. ${ }^{34-36}$

More recently, radioactive ligands have been developed to visualize aggregation of amyloid-beta $(\mathrm{A} \beta)$ peptides. The benzothiazole-derived $\left[{ }^{11} \mathrm{C}\right]$-Pittsburgh compound B PET ligand is an early example. It is retained in areas of the brain known to harbor amyloid plaques and increased levels can be demonstrated in some individuals with MCI, although abnormal retention can also occur in individuals with normal cognition. ${ }^{37,38}$

Routine SPECT images provide a qualitative visual map of cerebral perfusion and have been used for many years in the evaluation of patients with cognitive impairment as a surrogate marker of impaired neuronal metab- olism. ${ }^{39}$ Recently, compatible amyloid ligands have been developed. These ligands can be readily radiolabeled for in vivo imaging using SPECT or PET. These means provide the ability to quantiate amyloid plaque burdens in $\mathrm{AD}$ patients.

\section{BIOCHEMICAL MARKERS-SERUM AND CSF}

The most common measurements of the CSF, regarding $\mathrm{AD}$, focus on the tau protein and $\mathrm{A} \beta$-peptides. Neurofibrillar protein aggregates containing phosphorylated tau are one of the major hallmarks of $\mathrm{AD}$. In $\mathrm{AD}$, tau becomes abnormally phosphorylated, aggregates into paired helical filaments, and loses its ability to maintain the microtubule tracks. $A \beta$-peptides result from enzymatic breakdown of amyloid precursor protein by $\alpha-, \beta-$, and $\gamma$-secretases and make up the main part of amyloid deposits in $\mathrm{AD} .{ }^{40}$

Quantification of tau and $A \beta$ in the CSF represent the most intensively studied $\mathrm{BM}$ of $\mathrm{AD} .{ }^{41}$ Approximately $80 \%$ of patients who meet clinical criteria for $\mathrm{AD}$ have elevated levels of CSF tau. ${ }^{42}$ Autopsy studies confirmed this association and validated the relationship between high levels of CSF tau and a pathologic diagnosis of AD. ${ }^{43}$ Arai et al. ${ }^{44}$ have found that high CSF total tau (t-tau) could discriminate between memory-impaired individuals that later progressed to $\mathrm{AD}$, and those that did not convert. Other authors ${ }^{45}$ have shown that the absolute level of CSF tau in patients with AD did not correlate with the severity or duration of the dementia. Another line of recent evidence suggests that CSF tauprotein phosphorylated at threonine $231-\mathrm{CSF}$ p-tau 231 declines during the natural course of AD. Seventeen pharmacologically untreated patients with $\mathrm{AD}$ were followed up to 6 years with repeated serial CSF measurements. CSF p-tau 231 concentration, but not t-tau, decreased over time in $\mathrm{AD}$, independent of age. Rate of change was inversely correlated to the Mini Mental State Examination (MMSE) score at baseline and provided objective measure for the cognitive decline. ${ }^{46}$ These results suggest that CSF p-tau ${ }_{231}$ may have the potential to track $\mathrm{AD}$ progression, and may be a valuable tool to map effects of disease-modifying drugs on $\mathrm{AD}$ specific neurodegeneration.

$\mathrm{A} \beta_{(1-42)}$ is especially prone to fibrillization and disproportionally accumulates in extracellular lesions in $\mathrm{AD}$ brains, and was found to be reduced in the CSF of $\mathrm{AD}$ patients. ${ }^{42,43}$ The reduction in CSF A $\beta_{(1-42)}$ in AD has been hypothesized to reflect a deposition of the peptide in senile plaques, with lower levels diffusing to the CSF. Thus, according to Galasko et al., ${ }^{47}$ decrease in $A \beta(1-42)$ in $\mathrm{AD}$ is probably the most consistent $\mathrm{BM}$ finding. $\mathrm{A} \beta_{(1-}$ 42) alone showed a sensitivity of $78 \%$, and a specificity of $81 \%$, in distinguishing $\mathrm{AD}$ patients from elderly controls. However, studies correlating CSF A $\beta_{(1-42)}$ protein 
concentrations with cognitive performance in $\mathrm{AD}$ were, in part, contradictory. ${ }^{48-50}$ The potential value of $\mathrm{A} \beta_{(1-42)}$ protein during the course of $\mathrm{AD}$ progression should be further evaluated.

In addition to CSF BMs, studies by others have focused on plasma BMs. For example, the study by Ringman et al. ${ }^{51}$ provided preliminary support for a model in which BMs can predict disease progression in presymptomatic familial AD persons. Participants underwent evaluations for presenilin 1 or APP mutations, and cognitive evaluations with the Clinical Dementia Rating Scale (CDR). The data obtained in this study indicated that plasma $\mathrm{A} \beta_{42}$ is elevated in familial $\mathrm{AD}$ mutations carriers and that this level may decrease with the cognitive decline of disease progression prior to the development of dementia. These results are in conflict with those found by Hulstaert et al. ${ }^{49}$

\section{BIOMARKERS FOR PARKINSON'S DISEASE}

PD may be the second most common neurodegenerative disease after $\mathrm{AD}$, featuring an incidence that increases with age and a higher prevalence throughout the male population. The cardinal symptoms of PD include bradykinesia, hypokinesia or akinesia, muscular rigidity and resting tremor, often progressing to postural instability, gait problems, and freezing. Although attracting considerably less attention, several nonmotor symptoms related to autonomic disturbances, such as hypotension, constipation, bladder and thermoregulatory dysfunctions, and sleep disturbances are manifested. PD may be associated with olfactory dysfunction, depression and anxiety, together with the onset of cognitive deficits and dementia as the disease progresses. ${ }^{52}$

The pathological hallmark of PD is represented by degeneration of the nigrostriatal dopaminergic neurons associated to the presence of intracytoplasmatic inclusions of ubiquitin and alpha-synuclein ( $\alpha$-synuclein) denominated Lewy bodies. It is interesting to note that the motor symptoms characterizing the disease are manifested once degeneration of the dopaminergic nigrostriatal pathway has reached at least 70 to $80 \%$. Numerous recent studies have demonstrated the involvement of several other neurotransmitters, including acetylcholine, noradrenaline, glutamate, and adenosine.

$\mathrm{PD}$ is a multifactorial disease, and several factors related to genes, age, sex, and environment may increase the risk of contracting the disease. The most well-established hypothesis concerning the pathogenesis of PD suggests the involvement of a cascade, or cascades, of neurotoxic events, acting in parallel and/or in a sequential chain eventually leading to the demise of the melanin-containing dopaminergic neurons of the SN pars compacta. This cascade includes altered protein degradation, excessive iron accumulation in the SN pars compacta, oxidative stress, glutamatergic excitotoxicity, mitochondrial (complex I deficiency), and ubiquitin-proteasome system dysfunction, leading to abnormal protein folding and aggregation, inflammation, and apoptosis. ${ }^{53}$

To date, standard clinical diagnosis has been based on the application of clinical tools, such as the Unified Parkinson's Disease Rating Scale, the Hoehn and Yahr disability test and neuroimaging assessment. No clinical diagnostic test based on biochemical analysis of blood or CSF is as available yet. Thus, there is hope that BMs will help diagnose symptomatic and presymptomatic disease stages.

\section{PREDICTIVE BEHAVIORAL CLINICAL TESTS}

Symptoms including sleep disturbances, mood disorders, and olfactory and autonomic dysfunctions (e.g., constipation) have been identified during both the premotor and motor symptomatic phase of the disease. Accordingly, the presence of these dysfunctions may contribute to the clinical diagnosis of PD. The schematic in FIG. 2 represents the different phases of the disease, from onset to diagnosis.

Presence of sleep disturbance, with reduced rapid eye movements (REM) sleep, and frequent and prolonged waking throughout the night, is commonly associated with PD. REM sleep behavior disorder in patients affected by PD is closely related to incipient cognitive impairment, whereas quantitative motor testing, olfactory deficits, and autonomic symptoms are not. ${ }^{54,55} \mathrm{Re}$ duced REM sleep latency (time from sleep onset to the first REM episode) was found in a high proportion in PD patients; however, its incidence was significantly higher in depressed patients, suggesting that differences in latency may be related to depression rather than PD.

Depressed mood is often associated with PD, and several recent studies have confirmed that depression in the PD patient is a major determinant of quality of life. ${ }^{56}$ Depression in PD might be associated with a specific loss of dopamine (DA), serotonin and noradrenaline innervation in the limbic system. ${ }^{57}$ Interestingly, several studies have suggested that depression in the PD patient is associated with a more rapid deterioration in cognitive function. $^{56}$

Olfactory deficits reported in PD are believed to represent a sensitive marker of the disease, which could allow an early diagnosis and help in differentiating idiopathic PD from other diseases with features of Parkinsonism. The diagnostic accuracy of olfactory testing in differentiating PD from other disorders is however insufficient to justify routine clinical use. ${ }^{58}$ Olfactory dysfunctions are often measured by means of the University of Pennsylvania Smell Identification Test. Major deficit in olfactory judgment is present in PD, suggesting that this deficit is associated with disruption of olfactory areas situated in the temporal lobes and the prefrontal 


\section{Onset}

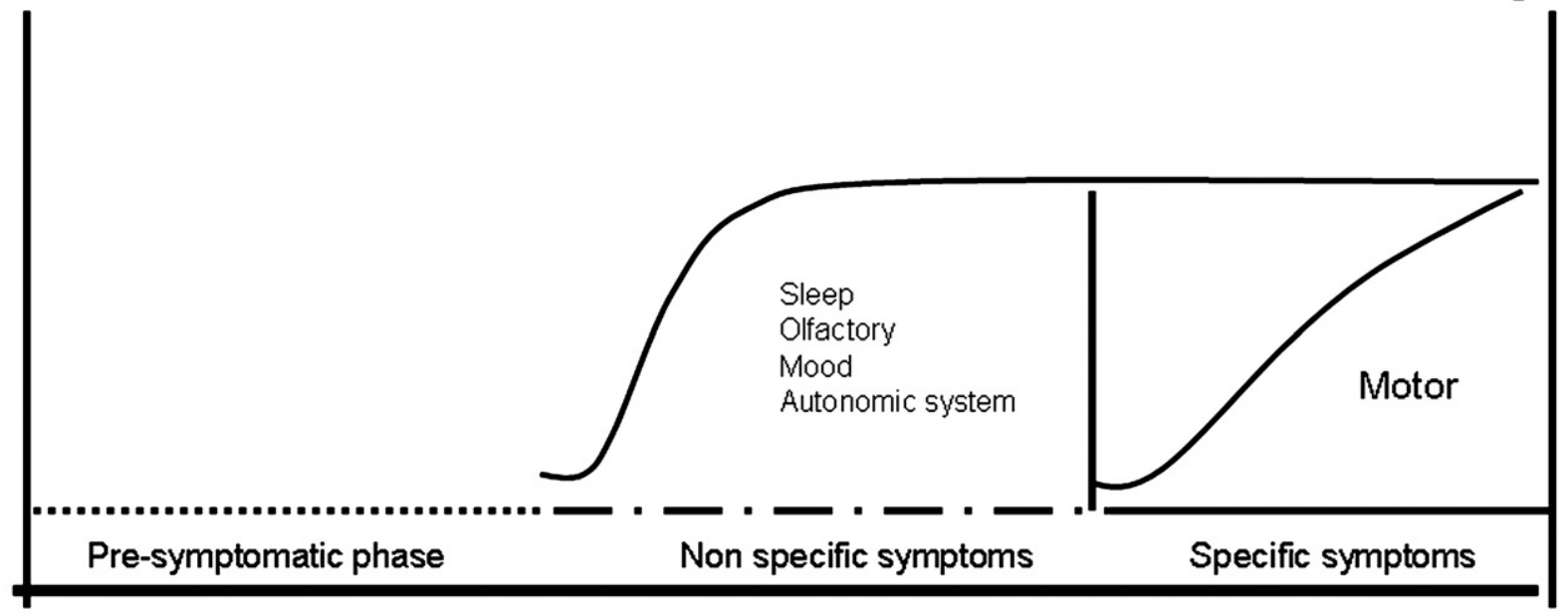

\section{Pre-diagnostic phase}

FIG. 2. Pre-diagnostic phase of Parkinson's disease (PD). Cerebral dopamine depletion, the hallmark of PD, develops slowly, and gradually progresses over years (pre-symptomatic phase). Before cardinal motor symptoms of PD appear, several nonmotor symptoms are present (nonspecific symptoms). At present several symptoms, including sleep disturbances, mood disorders, and olfactory and autonomic dysfunctions (e.g., constipation) have been identified during the pre-motor symptomatic phase. The presence of these dysfunctions may contribute toward an early clinical diagnosis of PD. These nonspecific symptoms persist in the motor phase when the cardinal symptoms of PD: bradykinesia, hypokinesia and akinesia, muscular rigidity, resting tremor, postural instability, gait problems, and freezing appear (more specific symptoms).

cortex ${ }^{59}$ although olfactory bulb changes may have an important role.

Diagnostic test battery. Montgomery et al. ${ }^{60} \mathrm{de}-$ veloped a behavioral diagnostic test battery to provide early diagnosis of PD. The test battery incorporates tests for motor, olfaction, and mood evaluation. As previously mentioned, symptoms of olfactory dysfunction and depression often antedate the diagnosis of PD; addition of the wrist flexion and extension movement test provided a test battery featuring high specificity (88\%) and sensitivity rate $(69 \%)$, not previously achieved by any test alone. The latter battery test has been successfully applied in the prospective identification of as yet undiagnosed patients with signs suggestive of PD who were subsequently diagnosed as affected by the disease. ${ }^{61}$

MCI. Based on findings reported in several studies on PD featuring dementia and dementia with Lewy bodies (DLB), Troster ${ }^{62}$ performed a critical evaluation to determine whether MCI may constitute an early symptom of PD with dementia, and whether it may prove possible to differentiate between PD with dementia, or DLB. The conclusions drawn in the review presently underline how the data available do not allow an adequate evaluation of the use of MCI, which for PD mainly concerns executive functions as a predictive test for PD with dementia. However, the author acknowledged that to date scarce attention has been devoted to cognitive impairment and the role of depression on the latter. Moreover, DLB and PD with dementia, although very similar from a pathological point of view, should be viewed as two separate entities. ${ }^{62}$

Autonomic dysfunctions. These include orthostatic dizziness, constipation, bladder dysfunction, pain, erectile dysfunction, and hyperhidrosis are associated with PD and appear as early symptoms prior to the onset of motor disorders. A new diagnostic strategy using skin biopsy, as developed by Sommer and Lauria, ${ }^{63}$ and applied to PD patients by Rossi et al., ${ }^{64}$ suggests that small peripheral fibers may be reduced in PD patients. Analysis of small caliber sensory nerves might therefore represent a suitable approach for use in the characterization of early autonomic dysfunctions in PD.

\section{BIOCHEMICAL MARKERS}

As previously mentioned, no laboratory tests for use in the detection of blood or CSF BMs correlating with sporadic forms of PD are currently available. We present here a series of BM tests of the clinical application of which is advised, together with other tests already applied in preliminary form, for the previously mentioned purpose.

A pioneer study performed by Harrington and Merril, ${ }^{65}$ described differences in CSF protein pattern in PD patients compared to healthy controls. Subsequently, additional studies performed on lymphocytes or platelets of PD patients have detected subtle abnormalities in DA signaling ${ }^{66-68}$ or mitochondrial functions, ${ }^{69-72}$ which con- 
stitute hallmarks of PD. None of the latter abnormalities, however, has reached the phase of clinical screening.

Activation of glial cells has been consistently found in the brain of patients affected by PD. The variations in prostaglandin D synthase isoforms described by Harrington et al..$^{73}$ are of great interest, because this reflects the pathophysiology of meninges and glia, which are a source of prostaglandin $\mathrm{D}$. The reported reduction of the prostaglandin D synthase isoforms variant 3 and 4 in CSF of PD patients, measured through two-dimensional gel electrophoresis and mass spectrometry, correlated to changes described in glial cells by several studies, suggesting the suitability of these isoforms as candidate diagnostics markers having a predictive value.

An unexpected, possible BM, linked to a reduced risk of $\mathrm{PD}$, is the concentration of serum urate, the main end-product of purine metabolism. ${ }^{74,75}$ Moreover, serum urate levels inversely correlate with progression of the disease. ${ }^{76}$ Urate exerts an antioxidant efficacy comparable to that displayed by ascorbate; accordingly, the high levels registered may act as a defense against oxidative damage, the main acknowledged cause of PD. Urate is implicated in cerebrovascular diseases; therefore, rather than being used as a neuroprotective agent, its blood levels may serve as an indirect marker of neuroprotection in neurodegenerative diseases, such as PD. In addition, urate may be of use in neuroprotective trials as an index of disease progression. ${ }^{76}$

Another interesting field of potentially useful BMs for PD is the differential expression of complement proteins. ${ }^{77}$ Several types of unidentified proteins present in blood serum have been studied by means of quantitative two-dimensional gel electrophoresis as BMs for use in the diagnosis of neurodegenerative diseases. ${ }^{77,78}$ By using this technique, 34 different serum proteins have been identified in PD and amyotrophic lateral sclerosis, nine of which belong to the complement system. Indeed, differences have been reported between the components of complement $\mathrm{C} 3 \mathrm{c}$ and complement factor $\mathrm{H}$ and $\mathrm{B}$ in affected subjects compared to controls. Differential expression of complement proteins may represent potentially useful BMs for amyotrophic lateral sclerosis and PD. ${ }^{79}$ These findings lend further support to evidence suggesting that inflammatory processes involving complement cascade elements may be involved in the onset of PD.

Using CSF of PD patients, Zhang et al. ${ }^{80}$ reported on the first large scale proteomic experiment, the presence of novel BMs for PD and AD. Of these BMs, however, only $\mathrm{A} \beta_{1-42}$ and tau proteins displayed different levels among controls, $\mathrm{AD}$ and $\mathrm{PD}$, and between $\mathrm{AD}$ and $\mathrm{PD}$ as expected. In contrast, the remainders (i.e, $\beta$ DNF, IL-8, vitamin-D binding protein, $\beta_{2}$-microbulin, haptoglobin, and apolipoprotein A-I, A-II, and E) were significantly different from healthy controls, but did not differ between PD and AD.

\section{GENETIC MARKERS}

Parkinsonism has long been viewed as a "sporadic" disorder, although a familial component was noted over a century ago. ${ }^{81,82}$ It was not until the last decade that the contribution of genetics to familial forms of PD became more generally acknowledged. At present, 12 genomic chromosomal regions and eight pathogenic mutations in genes unequivocally linked to familial PD, such as autosomal dominant and autosomal recessive PD, have been identified. ${ }^{83}$ The identification of single mutated genes linked to heritable forms of PD has further enlightened our understanding of its etio-pathophysiology in the non-genetic cases by providing potential molecular pathways that may help in the early identification of the disease and its treatment.

Interestingly, DJ-1 (PARK7) and $\alpha$-synuclein (SNCA), two critical interacting proteins, displayed modifications in CSF, serum, or plasma levels in sporadic PD. Lower CSF levels of $\alpha$-synuclein have been observed in neurological diseases with primary synucleinopathy, such as DLB and PD ${ }^{84}$ whereas plasma levels were increased. ${ }^{85}$ These studies have described interesting methods for use in the quantification of $\alpha$-synuclein in biological fluids in diseases characterized by synucleinopathy. No differences were reported in serum DJ-1 levels measured in PD patients and healthy controls in a study performed by Maita et al., ${ }^{86}$ whereas other studies performed in CSF or plasma reported higher DJ-1 levels in PD patients than controls. ${ }^{87,88}$ Moreover, while upregulation of CSF DJ-1 levels in early stages of PD was more marked compared to levels observed in advanced stages and in controls, plasma DJ-1 levels appeared to be higher in advanced disease. Likewise, these studies revealed similar increases in PD and in neurological diseases with primary synucleinopathy. ${ }^{87,88}$ How DJ- 1 and $\alpha$-synuclein in biological fluids may be of use as BMs in differentiating PD from other neurological disorders remains to be established.

For the scope of BMs in neurodegenerative diseases, it should be borne in mind how clinical proteomic and consequently predictive BMs are strongly influenced by pre-analytical factors, such as time or condition of storage. ${ }^{89}$ Accumulation of altered proteins and impaired protein clearance may underlie the onset of PD. Recent advances made in methodological procedures used in the study of PD-associated proteins involving the systematic combined use of BMs may lead to findings capable of providing a crucial contribution to enhancing diagnosis of PD. 


\section{NEUROIMAGING MARKERS}

Imaging PD involves either detecting alterations in brain structure or examining functional changes in brain metabolic systems. In PD, degeneration of the dopaminergic system is accompanied by a cholinergic, noradrenergic, and serotonergic dysfunction. Function of these dopaminergic and nondopaminergic systems can be imaged with PET and SPECT and correlated with motor and nonmotor disturbances. Dopa decarboxylase activity at DA terminals and DA turnover can both be measured with ${ }^{18} \mathrm{~F}$-dopa PET. Presynaptic DA transporters (DATs) can be followed with PET and SPECT tracers, such as ${ }^{123} \mathrm{I}-2 \beta$-carbomethoxy-3 $\beta$-(4-iodophenyl)-N-(3iodophenyl)tropane while vesicle monoamine transporter density in DA terminals can be examined with ${ }^{11} \mathrm{C}$ dihydrotetrabenazine PET. Measurements of DA terminal function can sensitively detect DA deficiency in both symptomatic patients and individuals at risk for parkinsonian syndromes, ${ }^{90}$ but have poor specificity for discriminating between typical and atypical PD. On the other hand, measurements of glucose metabolism with $\left[{ }^{18} \mathrm{~F}\right]$-fluoro-deoxy-D-glucose PET can be very helpful as normal or raised levels were found in the lentiform nucleus of PD, but levels were reduced in multiple system atrophy and progressive supranuclear palsy. ${ }^{91}$

MRI and transcranial sonography can reveal brain structural changes, such as volumetric reduction and hyperechogenicity of certain midbrain and striatal areas in patients with PD. ${ }^{92}$ Recent structural neuroimaging studies applying noninvasive transcranial sonography have shown that more than $90 \%$ of PD patients show hyperechogenicity of the SN compared to healthy individu$\mathrm{als}^{93}$ and this could be related in part to an increased iron content of the SN. ${ }^{94}$ Even more, in young healthy adults who presented increased echogenicity of the SN, a functional impairment of the nigrostriatal system was evident. Brain areas responsible for motor functions (extrapyramidal regions) appear to have more iron than nonmotor-related regions, which might explain why movement disorders are commonly associated with iron imbalance. ${ }^{95,96}$ This preferential iron localization might directly increase the susceptibility of neurons and surrounding cells to toxins and inflammatory processes. High concentrations of reactive iron contributes to the enhanced generation of reactive oxygen species, which in turn can increase oxidative stress and protein aggregation, including the aggregation of $\alpha$-synuclein and $\mathrm{A} \beta$, two major contributors of $\mathrm{PD}$ and $\mathrm{AD}$ pathology, respectively.

Transcranial sonography and MRI might be particularly valuable for revealing a susceptibility to PD that can be detected early in life prior to the clinical manifestation of PD, although they correlate less well with either clinical status or loss of DA terminal function in the striatum. By contrast, PET and SPECT measurements of DA terminal function correlate significantly with clinical disability. ${ }^{90}$

In summary, although the available BMs for PD are promising, none of them can predict the disease with a high percentage of certainty, thus it seems, like in $\mathrm{AD}$, the best diagnosis will require a combination of markers and clinical assessment.

\section{TRANSCRIPTOMIC SIGNATURES FOR EVALUATION OF CLINICAL EFFICACY OF NEUROPROTECTIVE DISEASE-MODIFYING THERAPY}

It is well accepted that PD is a multisystem disorder involving both brain and peripheral areas, in which predisposed neuronal cell types in specific regions of the human peripheral, enteric, and CNS become progressively involved. ${ }^{97}$ Accordingly, the damage to SN pars compacta occurs later in the course of the disease, whereas other brain areas and peripheral tissues are initially affected at the pre-symptomatic phase of the disease. Currently, available measures of neuroprotection are indirect and comprise functional imaging and clinical outcomes, however, which do not always correlate. This limits the ability to test neuroprotective drugs with disease-modifying ability. Clearly, the identification of suitable valid BMs that can be monitored from preclinical stages in asymptomatic "at-risk" subjects, will allow the capturing of high-risk individuals in the valuable therapeutic window of about 5 years before the appearance of clinical signs. In addition, specific BMs will permit assessment of the potential activity of specific therapies and development of CNS target-directed personalized drugs.

\section{Gene expression profiling of sporadic PD SN}

The current unsatisfactory situation with early diagnosis and available measures of neuroprotection in PD has prompted researchers to look for new approaches using smart, advanced biotechnology. During the course of the past decade, we are witnessing the advent of high throughput gene and protein techniques, which are becoming especially relevant in the neuroprotective field for aging and neurodegenerative diseases, because they can provide important targets to develop disease-modifying therapies that may have an impact. Recent high microarray-assisted large-scale transcriptomic studies in human postmortem SN from sporadic PD have revealed significant dysregulation of genes from biological processes linked to previously established neurodegenerative mechanisms both in sporadic and hereditary PD. These include protein aggregation, mitochondrial dysfunction, oxidative stress, cell cycle, vesicle trafficking, synaptic transmission, DA metabolism, and cell adhe- 


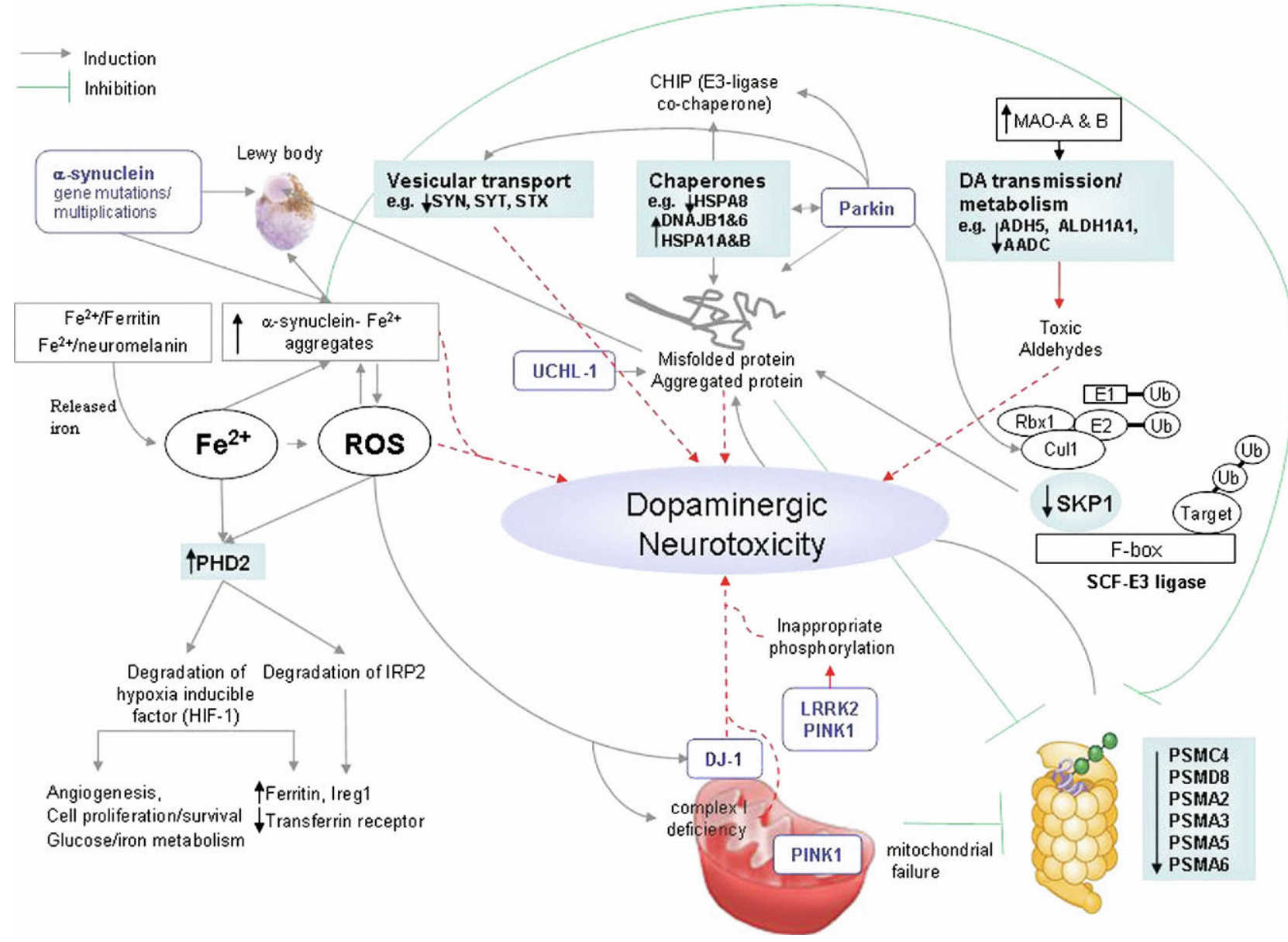

FIG. 3. Molecular cascades of dopaminergic neurotoxicity in human parkinsonian substantia nigra (SN). Gene expression profiling studies in human postmortem Parkinson's disease (PD) SN, share the identification of similar functional classes, including DA metabolism and transmission, vesicular transport, cytoskeleton, and protein handling/degradation. For instance, decreases in Cullin, F-box (taken from ${ }^{54}$ ) SKP1A (a ubiquitin E3 ligase component), chaperones, and proteasome subunits can lead to a general impairment in the normal degradation of wide arrays of proteins, which may include activators of cell cycle, $\alpha$-synuclein, parkin, iron regulatory protein 2 (IRP2), hypoxia inducible factor (HIF). Their decline can cause evasion of proteins directed to degradation. Aldehyde dehydrogenase $(A L D H)$ and alcohol dehydrogenase (ADH) are involved in the degradation of aldehyde derivatives of dopamine (DA), generated by the action of monoamine oxidase (MAO)-A and MAO-B. These highly reactive, neurotoxic aldehydes can accumulate in case of decreased levels of ALDH and $\mathrm{ADH}$ and promote neuronal death. Neurodegeneration can also result from impaired iron transport across the blood-brain barrier, release of iron from ferritin or neuromelanin, generation of reactive hydroxyl radicals (OH $\bullet$ ) and induction of OS in the SNpc DA-containing neurons. In addition, free (labile) iron can cause aggregation of $\alpha$-synuclein and degradation of IRP2 via activation of prolyl-4-hydroxylase (PHD2, encoded by EGLN1), which is a key iron and OS sensor. This in turn results in proteasomal degradation of HIF and IRP2, with subsequent decrease in cell survival/proliferation, glucosem and iron metabolism genes. The various familial PD associated genes (e.g., $\alpha$-synuclein, parkin, DJ-1, LRRK2) have been intercalated at different molecular pathways to illustrate potential cross-talks sites common to sporadic and hereditary PD. PSMC4 = proteasome (prosome, macropain) 26S subunit, ATPase $1 / 4 ; \mathrm{PSMA}=$ proteasome (prosome, macropain) subunit; $\mathrm{ADLH} 1 \mathrm{~A} 1$ = aldehyde dehydrogenase 1 family, member $\mathrm{A} 1 ; \mathrm{ADH} 5=\mathrm{alcohol}$ dehydrogenase 5; AADC = aromatic amino acid decarboxylase; SYN = synapsin; SYT = synaptotagmin; STX = syntaxin; HSPA = heat shock protein A; DNAJB = DnaJ (Hsp40) homolog, subfamily B; CHIP = carboxyl-terminus of Hsc70 interacting protein; SKP1A = S-phase kinase-associated protein 1A(p19A); SCF = SKP, Cullin, F-box protein; ROS = reactive oxygen species.

sion/cytoskeleton maintenance. The selected number of candidate genes identified by the various research groups may offer clues for potential gene intersections or crosstalks along the dopaminergic neurodegenerative cascade. FIG. 3 summarizes relevant molecular cascades of neurotoxicity in human parkinsonian $\mathrm{SN}$ as unraveled by the different research groups.

Interestingly, all the gene expression profiling studies conducted so far in the SN of PD patients reported a dysfunction in vesicle trafficking and synaptic trans- mission functional group, such as syntaxin-1 (STX1), synaptotagmin-1 (SYT1) and synapsin 1 and 2 $(S Y N 1,2)$. It is possible to speculate that these proteins may functionally overlap in a common pathway of cytoskeletal maintenance and intracellular vesicle transport. Also, all the studies revealed a consistent elevation in heat-shock proteins/stress genes, such as the endoplasmic reticulum protein homocysteine-inducible, endoplasmic reticulum stress-inducible, ubiquitin-like domain member 1 (HERPUD1), and DnaJ 
(Hsp40) homologue subfamily $\mathrm{B}$, members 1 and 5 (DNAJB 1/5), heat shock proteins $\mathrm{A} 1 \mathrm{~A}$ and $\mathrm{A} 1 \mathrm{~B}$ (HSPAIA, HSPAlB), and heat shock $27 \mathrm{kDa}$ protein 1 $(H S P B 1)^{53}$.

\section{Blood expression profiling of sporadic PD}

Currently, there is no laboratory blood test that can diagnose PD, making the detection of individuals at risk or at earliest stages of PD practically impossible. For a BM to be clinically useful, noninvasive detection in accessible peripheral tissue is desirable. Blood lymphocytes may offer valuable surrogate markers for neuropsychiatric disorders, as they share significant gene expression similarities to more inaccessible CNS tissues. ${ }^{98}$ Despite the differences in embryonic lineage and biological function between blood and brain, this correlation could be explained, in part, by the fact that blood cells circulate through many different body tissues and thus, may be exposed to the same toxic atmosphere surrounding, for instance, the dopaminergic neurons in the SN pars compacta. Under these circumstances, the blood cells can alter their transcriptome in response to pathological perturbations, acting as "sentinels," as recently proposed. ${ }^{99}$ For example, a recent high throughput microarray gene analysis applied concomitantly to peripheral blood cells and dorsolateral prefrontal cortex from schizophrenia patients, has revealed a concurrent validation of a subset of genes, which may be considered reliable candidate risk genes and BMs of the disease. ${ }^{100}$ Regardless of which disease is investigated, the most precise results will be obtained for a carefully selected subset of genes that show similar expression profiles across blood and CNS tissues. ${ }^{98}$ Recently, Scherzer et al. ${ }^{101,102}$ have published the results of a transcriptomewide scan in whole blood tissue from early-staged PD individuals and age-matched controls. The authors reported under-expression of the HSP70-interacting protein ST13 and coexpression and coinduction of SNCA gene with critical enzymes of heme metabolism, in patients with PD versus healthy individuals. These findings suggest that blood and neuronal cells might have a common regulatory mechanism for gene expression. Analogous to drug development and approval, novel candidate BMs for PD risk assessment will require additional cohort studies including larger PD population size, adequately selected on the basis of disease onset, date of medication initiation, and follow-up program, as well as controls with other neurodegenerative diseases.

As with any clinical assay, standardized measures for blood sample collection (e.g., stabilization of blood transcriptome) and shipping is of utmost importance before the process of RNA extraction to reduce pre-analytical variability. ${ }^{99}$

\section{CONCLUSIONS}

The development of neuroprotective strategies is greatly hindered by the lack of markers capable of serving as objective surrogate endpoints for clinical trials testing these treatments. Several experimental methods have been used to identify BMs in early disease stage, most studies have examined only a single BM, thus preventing assessment of their relative sensitivities and specificities. Although these BMs are promising, none of them can predict $\mathrm{AD}$ or PD with $100 \%$ confidence nor provide a clear delineation of subgroups at risk and thus, a combination of markers may be necessary. This seems difficult, especially because they are costly, invasive, or unsuitable for broad application.

The rapid growth of molecular biology and laboratory technology has expanded to the point at which the application of technically advanced BMs becomes broadly available and applicable. Such BMs and the additional occurrence of complementary markers will be useful in mapping disease pathogenesis and defining subjects at risk for $\mathrm{AD}$ and $\mathrm{PD}$. Of particular importance, they would be able to determine the clinical efficacy of novel disease-modifying (neuroprotective) strategies.

\section{REFERENCES}

1. Fratiglioni L, Launer LJ, Andersen K, et al. Incidence of dementia and major subtypes in Europe: a collaborative study of population-based cohorts. Neurologic Diseases in the Elderly Research Group. Neurology 2000;54:S10-S15.

2. Lobo A, Launer LJ, Fratiglioni L, et al. Prevalence of dementia and major subtypes in Europe: A collaborative study of population-based cohorts. Neurologic Diseases in the Elderly Research Group. Neurology 2000;54:S4-S9.

3. Graham JE, Rockwood K, Beattie BL, et al. Prevalence and severity of cognitive impairment with and without dementia in an elderly population. Lancet 1997;349:1793-1796.

4. Poewe W, Wenning G. The differential diagnosis of Parkinson's disease. Eur J Neurol 2002;9 Suppl 3:23-30.

5. Galasko D, Hansen LA, Katzman R, et al. Clinical-neuropathological correlations in Alzheimer's disease and related dementias. Arch Neurol 1994;51:888-895.

6. Hulette C, Nochlin D, McKeel D, et al. Clinical-neuropathologic findings in multi-infarct dementia: a report of six autopsied cases. Neurology 1997;48:668-672.

7. Lyketsos CG, Szekely CA, Mielke MM, Rosenberg PB, Zandi PP. Developing new treatments for Alzheimer's disease: the who, what, when, and how of biomarker-guided therapies. Int Psychogeriatr 2008;20:871-889.

8. Kawas C, Gray S, Brookmeyer R, Fozard J, Zonderman A. Agespecific incidence rates of Alzheimer's disease: the Baltimore Longitudinal Study of Aging. Neurology 2000;54:2072-2077.

9. Youdim MB. The path from anti Parkinson drug selegiline and rasagiline to multifunctional neuroprotective anti Alzheimer drugs ladostigil and m30. Curr Alzheimer Res 2006;3:541-550.

10. McKhann G, Drachman D, Folstein M, Katzman R, Price D, Stadlan EM. Clinical diagnosis of Alzheimer's disease: report of the NINCDS-ADRDA Work Group under the auspices of Department of Health and Human Services Task Force on Alzheimer's Disease. Neurology 1984;34:939-944.

11. Sunderland T, Hampel H, Takeda M, Putnam KT, Cohen RM. Biomarkers in the diagnosis of Alzheimer's disease: are we ready? J Geriatr Psychiatry Neurol 2006;19:172-179.

12. Clark CM, Davatzikos C, Borthakur A, et al. Biomarkers for early detection of Alzheimer pathology. Neurosignals 2008;16:11-18. 
13. Halperin I, Korczyn AD. [Mild cognitive impairment (MCI): characteristics, risk factors and prevention]. Harefuah 2006;145: 229-234, 243.

14. Hänninen T, Hallikainen M, Tuomainen S, Vanhanen M, Soininen H. Prevalence of mild cognitive impairment: a populationbased study in elderly subjects. Acta Neurol Scand 2002;106: $148-154$.

15. Bertram L, Tanzi RE. The current status of Alzheimer's disease genetics: what do we tell the patients? Pharmacol Res 2004;50: 385-396.

16. Tanzi RE, Bertram L. New frontiers in Alzheimer's disease genetics. Neuron 2001;32:181-184.

17. Mulder C, Scheltens P, Visser JJ, van Kamp GJ, Schutgens RB. Genetic and biochemical markers for Alzheimer's disease: recent developments. Ann Clin Biochem 2000;37 ( Pt 5):593-607.

18. Otto M, Lewczuk P, Wiltfang J. Neurochemical approaches of cerebrospinal fluid diagnostics in neurodegenerative diseases. Methods 2008;44:289-298.

19. Corder EH, Saunders AM, Strittmatter WJ, et al. Gene dose of apolipoprotein E type 4 allele and the risk of Alzheimer's disease in late onset families. Science 1993;261:921-923.

20. Resnick SM, Goldszal AF, Davatzikos C, et al. One-year age changes in MRI brain volumes in older adults. Cereb Cortex 2000; $10: 464-472$.

21. Sullivan EV, Pfefferbaum A, Adalsteinsson E, Swan GE, Carmelli D. Differential rates of regional brain change in callosal and ventricular size: a 4-year longitudinal MRI study of elderly men. Cereb Cortex 2002;12:438-445.

22. Chetelat G, Baron JC. Early diagnosis of Alzheimer's disease: contribution of structural neuroimaging. Neuroimage 2003;18: 525-541.

23. Borthakur A, Wheaton AJ, Gougoutas AJ, et al. In vivo measurement of T1rho dispersion in the human brain at 1.5 tesla. J Magn Reson Imaging 2004;19:403-409.

24. Klunk WE, Panchalingam K, Moossy J, McClure RJ, Pettegrew JW. N-acetyl-L-aspartate and other amino acid metabolites in Alzheimer's disease brain: a preliminary proton nuclear magnetic resonance study. Neurology 1992;42:1578-1585.

25. Kwo-On-Yuen PF, Newmark RD, Budinger TF, Kaye JA, Ball MJ, Jagust WJ. Brain N-acetyl-L-aspartic acid in Alzheimer's disease: a proton magnetic resonance spectroscopy study. Brain Res 1994;667:167-174.

26. Jessen F, Block W, Traber F, et al. Proton MR spectroscopy detects a relative decrease of $\mathrm{N}$-acetylaspartate in the medial temporal lobe of patients with AD. Neurology 2000;55:684-688.

27. Schuff N, Capizzano AA, Du AT, et al. Selective reduction of $\mathrm{N}$-acetylaspartate in medial temporal and parietal lobes in AD. Neurology 2002;58:928-935.

28. Kantarci K, Jack CR, Jr., Xu YC, et al. Regional metabolic patterns in mild cognitive impairment and Alzheimer's disease: A 1H MRS study. Neurology 2000;55:210-217.

29. MacKay S, Ezekiel F, Di Sclafani V, et al. Alzheimer disease and subcortical ischemic vascular dementia: evaluation by combining MR imaging segmentation and H-1 MR spectroscopic imaging. Radiology 1996;198:537-545.

30. Kantarci K, Jack CR Jr, Xu YC, et al. Regional metabolic patterns in mild cognitive impairment and Alzheimer's disease: a 1H MRS study. Neurology 2000;55:210-217.

31. Foster NL, Heidebrink JL, Clark CM, et al. FDG-PET improves accuracy in distinguishing frontotemporal dementia and Alzheimer's disease. Brain 2007;130:2616-2635.

32. Silverman DH, Small GW, Chang CY, et al. Positron emission tomography in evaluation of dementia: Regional brain metabolism and long-term outcome. JAMA 2001;286:2120-2127.

33. Jagust W. Molecular neuroimaging in Alzheimer's disease. NeuroRx 2004;1:206-212.

34. Johnson KA, Holman BL, Rosen TJ, Nagel JS, English RJ, Growdon JH. Iofetamine I 123 single photon emission computed tomography is accurate in the diagnosis of Alzheimer's disease. Arch Intern Med 1990;150:752-756.

35. Eberling JL, Jagust WJ, Reed BR, Baker MG. Reduced temporal lobe blood flow in Alzheimer's disease. Neurobiol Aging 1992; 13:483-491.
36. Johnson KA, Jones K, Holman BL, et al. Preclinical prediction of Alzheimer's disease using SPECT. Neurology 1998;50:1563-1571.

37. Archer HA, Edison P, Brooks DJ, et al. Amyloid load and cerebral atrophy in Alzheimer's disease: an 11C-PIB positron emission tomography study. Ann Neurol 2006;60:145-147.

38. Engler H, Forsberg A, Almkvist O, et al. Two-year follow-up of amyloid deposition in patients with Alzheimer's disease. Brain 2006;129:2856-2866.

39. Jagust W, Thisted R, Devous MD, Sr., et al. SPECT perfusion imaging in the diagnosis of Alzheimer's disease: a clinical-pathologic study. Neurology 2001;56:950-956.

40. Shoji M, Golde TE, Ghiso J, et al. Production of the Alzheimer amyloid beta protein by normal proteolytic processing. Science 1992;258:126-129.

41. Blennow K. Cerebrospinal fluid protein biomarkers for Alzheimer's disease. NeuroRx 2004;1:213-225.

42. Blennow K, Vanmechelen E, Hampel H. CSF total tau, Abeta42 and phosphorylated tau protein as biomarkers for Alzheimer's disease. Mol Neurobiol 2001;24:87-97.

43. Clark CM, Xie S, Chittams J, et al. Cerebrospinal fluid tau and beta-amyloid: how well do these biomarkers reflect autopsy-confirmed dementia diagnoses? Arch Neurol 2003;60:1696-1702.

44. Arai H, Nakagawa T, Kosaka Y, et al. Elevated cerebrospinal fluid tau protein level as a predictor of dementia in memoryimpaired patients. Alzheimer's Research 1997;3:211-213.

45. Schonknecht P, Pantel J, Hartmann T, et al. Cerebrospinal fluid tau levels in Alzheimer's disease are elevated when compared with vascular dementia but do not correlate with measures of cerebral atrophy. Psychiatry Res 2003;120:231-238.

46. Hampel H, Buerger K, Kohnken R, et al. Tracking of Alzheimer's disease progression with cerebrospinal fluid tau protein phosphorylated at threonine 231. Ann Neurol 2001;49:545-546.

47. Galasko D, Clark C, Chang L, Miller B, Green RC, Motter R, Seubert P. Assessment of CSF levels of tau protein in mildly demented patients with Alzheimer's disease. Neurology 1997; 48:632-635.

48. Samuels SC, Silverman JM, Marin DB, et al. CSF beta-amyloid, cognition, and APOE genotype in Alzheimer's disease. Neurology 1999;52:547-551.

49. Hulstaert F, Blennow K, Ivanoiu A, et al. Improved discrimination of AD patients using beta-amyloid(1-42) and tau levels in CSF. Neurology 1999;52:1555-1562.

50. Andreasen N, Minthon L, Davidsson P, et al. Evaluation of CSFtau and CSF-Abeta42 as diagnostic markers for Alzheimer disease in clinical practice. Arch Neurol 2001;58:373-379.

51. Ringman JM, Younkin SG, Pratico D, et al. Biochemical markers in persons with preclinical familial Alzheimer disease. Neurology 2008;71:85-92.

52. Obeso JA, Rodriguez-Oroz MC, Rodriguez M, et al. Pathophysiology of the basal ganglia in Parkinson's disease. Trends Neurosci 2000;23:S8-19.

53. Mandel S, Amit T, Kalfon L, Youdim MB. Applying transcriptomic and proteomic knowledge to Parkinson's disease drug discovery. Expert opinion on drug discovery 2007;2:1225-1240.

54. Vendette M, Gagnon JF, Decary A, et al. REM sleep behavior disorder predicts cognitive impairment in Parkinson's disease without dementia. Neurology 2007;69:1843-1849.

55. Postuma RB, Gagnon JF, Vendette M, Charland K, Montplaisir J. REM sleep behaviour disorder in Parkinson's disease is associated with specific motor features. J Neurol Neurosurg Psychiatry 2008;79:1117-1121.

56. Burn DJ. Beyond the iron mask: towards better recognition and treatment of depression associated with Parkinson's disease. Mov Disord 2002;17:445-454.

57. Remy P, Doder M, Lees A, Turjanski N, Brooks D. Depression in Parkinson's disease: loss of dopamine and noradrenaline innervation in the limbic system. Brain 2005;128:1314-1322.

58. McKinnon JH, Demaerschalk BM, Caviness JN, Wellik KE, Adler CH, Wingerchuk DM. Sniffing out Parkinson disease: can olfactory testing differentiate parkinsonian disorders? Neurologist 2007;13:382-385.

59. Hudry J, Thobois S, Broussolle E, Adeleine P, Royet JP. Evi- 
dence for deficiencies in perceptual and semantic olfactory processes in Parkinson's disease. Chem Senses 2003;28:537-543.

60. Montgomery EB, Jr., Koller WC, LaMantia TJ, et al. Early detection of probable idiopathic Parkinson's disease: I. Development of a diagnostic test battery. Mov Disord 2000;15:467-473.

61. Montgomery EB, Jr., Lyons K, Koller WC. Early detection of probable idiopathic Parkinson's disease: II. A prospective application of a diagnostic test battery. Mov Disord 2000;15:474-478.

62. Troster AI. Neuropsychological characteristics of dementia with Lewy bodies and Parkinson's disease with dementia: differentiation, early detection, and implications for "mild cognitive impairment" and biomarkers. Neuropsychol Rev 2008;18:103-119.

63. Sommer C, Lauria G. Skin biopsy in the management of peripheral neuropathy. Lancet Neurol 2007;6:632-642.

64. Rossi A, Giovenali P, Benvenuti M, Di Iorio W, Calabresi P. Skin biopsy: a new diagnostic tool for autonomic dysfunctions in Parkinson's disease? Lancet Neurol 2007;6:848-849; author reply 849.

65. Harrington MG, Merril CR. Two-dimensional electrophoresis and "ultrasensitive" silver staining of cerebrospinal fluid proteins in neurological diseases. Clin Chem 1984;30:1933-1937.

66. Nagai Y, Ueno S, Saeki Y, Soga F, Hirano M, Yanagihara T. Decrease of the D3 dopamine receptor mRNA expression in lymphocytes from patients with Parkinson's disease. Neurology 1996;46:791-795.

67. Barbanti P, Fabbrini G, Ricci A, et al. Increased expression of dopamine receptors on lymphocytes in Parkinson's disease. Mov Disord 1999;14:764-771

68. Caronti B, Antonini G, Calderaro C, et al. Dopamine transporter immunoreactivity in peripheral blood lymphocytes in Parkinson's disease. J Neural Transm 2001;108:803-807.

69. Parker WD, Jr., Boyson SJ, Parks JK. Abnormalities of the electron transport chain in idiopathic Parkinson's disease. Ann Neurol 1989;26:719-723.

70. Benecke R, Strumper P, Weiss H. Electron transfer complexes I and IV of platelets are abnormal in Parkinson's disease but normal in Parkinson-plus syndromes. Brain 1993;116 ( Pt 6): $1451-1463$.

71. Haas RH, Nasirian F, Nakano K, et al. Low platelet mitochondrial complex I and complex II/III activity in early untreated Parkinson's disease. Ann Neurol 1995;37:714-722.

72. Migliore L, Petrozzi L, Lucetti C, et al. Oxidative damage and cytogenetic analysis in leukocytes of Parkinson's disease patients. Neurology 2002;58:1809-1815.

73. Harrington MG, Fonteh AN, Biringer RG, AF RH, Cowan RP. Prostaglandin D synthase isoforms from cerebrospinal fluid vary with brain pathology. Dis Markers 2006;22:73-81.

74. Davis JW, Grandinetti A, Waslien CI, Ross GW, White LR, Morens DM. Observations on serum uric acid levels and the risk of idiopathic Parkinson's disease. Am J Epidemiol 1996;144: $480-484$.

75. Weisskopf MG, O'Reilly E, Chen H, Schwarzschild MA, Ascherio A. Plasma urate and risk of Parkinson's disease. Am J Epidemiol 2007;166:561-567.

76. Schwarzschild MA, Schwid SR, Marek K, et al. Serum urate as a predictor of clinical and radiographic progression in Parkinson disease. Arch Neurol 2008;65:716-723.

77. Goldknopf IL. Blood-based proteomics for personalized medicine: examples from neurodegenerative disease. Expert Rev Proteomics 2008;5:1-8.

78. Sheta EA, Appel SH, Goldknopf IL. 2D gel blood serum biomarkers reveal differential clinical proteomics of the neurodegenerative diseases. Expert Rev Proteomics 2006;3:45-62.

79. Goldknopf IL, Sheta EA, Bryson J, et al. Complement C3c and related protein biomarkers in amyotrophic lateral sclerosis and Parkinson's disease. Biochem Biophys Res Commun 2006;342: 1034-1039.
80. Zhang J, Sokal I, Peskind ER, et al. CSF multianalyte profile distinguishes Alzheimer and Parkinson diseases. Am J Clin Pathol 2008;129:526-529.

81. Gowers WR. A manual of diseases of the nervous system. Philadelphia: P. Blakiston; 1888. pp. 1027-1049.

82. Leroux P-D. Contribution à l'étude des causes de la paralysie agitante. (in French). Thèse de Paris, imprimeur de la faculté de médecine 1880

83. Rosner S, Giladi N, Orr-Urtreger A. Advances in the genetics of Parkinson's disease. Acta Pharmacol Sin 2008;29:21-34.

84. Mollenhauer B, Cullen V, Kahn I, et al. Direct quantification of CSF alpha-synuclein by ELISA and first cross-sectional study in patients with neurodegeneration. Exp Neurol 2008;213:315-325.

85. El-Agnaf OM, Salem SA, Paleologou KE, et al. Detection of oligomeric forms of alpha-synuclein protein in human plasma as a potential biomarker for Parkinson's disease. Faseb J 2006;20: 419-425.

86. Maita C, Tsuji S, Yabe I, et al. Secretion of DJ-1 into the serum of patients with Parkinson's disease. Neurosci Lett 2008;431:86-89.

87. Waragai M, Wei J, Fujita M, et al. Increased level of DJ-1 in the cerebrospinal fluids of sporadic Parkinson's disease. Biochem Biophys Res Commun 2006;345:967-972.

88. Waragai M, Nakai M, Wei J, et al. Plasma levels of DJ-1 as a possible marker for progression of sporadic Parkinson's disease. Neurosci Lett 2007;425:18-22.

89. Zetterberg H, Ruetschi U, Portelius E, et al. Clinical proteomics in neurodegenerative disorders. Acta Neurol Scand 2008;118:1-11.

90. Brooks DJ. Technology insight: imaging neurodegeneration in Parkinson's disease. Nat Clin Pract Neurol 2008;4:267-277.

91. Eckert T, Tang C, Eidelberg D. Assessment of the progression of Parkinson's disease: a metabolic network approach. Lancet Neurol 2007;6:926-932.

92. Berg D. Biomarkers for the early detection of Parkinson's and Alzheimer's disease. Neurodegener Dis 2008;5:133-136.

93. Berg D, Siefker C, Becker G. Echogenicity of the substantia nigra in Parkinson's disease and its relation to clinical findings. J Neurol 2001;248:684-689.

94. Berg D, Roggendorf W, Schroder U, et al. Echogenicity of the substantia nigra: association with increased iron content and marker for susceptibility to nigrostriatal injury. Arch Neurol 2002;59:999-1005.

95. Zecca L, Youdim MB, Riederer P, Connor JR, Crichton RR. Iron, brain ageing and neurodegenerative disorders. Nat Rev Neurosci 2004;5:863-873.

96. Koeppen AH. The history of iron in the brain. J Neurol Sci 1995;134(Suppl): 1-9.

97. Braak H, Muller CM, Rub U, et al. Pathology associated with sporadic Parkinson's disease-where does it end? J Neural Transm Suppl 2006:89-97.

98. Sullivan PF, Fan C, Perou CM. Evaluating the comparability of gene expression in blood and brain. Am J Med Genet B Neuropsychiatr Genet 2006;141B:261-268.

99. Burczynski ME, Dorner AJ. Transcriptional profiling of peripheral blood cells in clinical pharmacogenomic studies. Pharmacogenomics 2006;7:187-202.

100. Glatt SJ, Everall IP, Kremen WS, et al. Comparative gene expression analysis of blood and brain provides concurrent validation of SELENBP1 up-regulation in schizophrenia. Proc Natl Acad Sci U S A 2005;102:15533-15538.

101. Scherzer CR, Eklund AC, Morse LJ, et al. Molecular markers of early Parkinson's disease based on gene expression in blood. Proc Natl Acad Sci U S A 2007;104:955-960.

102. Scherzer CR, Grass JA, Liao Z, et al. GATA transcription factors directly regulate the Parkinson's disease-linked gene alphasynuclein. Proc Natl Acad Sci U S A 2008;105:10907-10912. 